\title{
The relation between syllable number and visual complexity in the acquisition of word meanings
}

\author{
MICHAEL H. KELLY \\ University of Pennsylvania, Philadelphia, Pennsylvania \\ and \\ KEN SPRINGER and FRANK C. KEIL \\ Cornell University, Ithaca, New York
}

\begin{abstract}
Four experiments were conducted to explore the correlation between syllable number and visual complexity in the acquisition of novel words. In the first experiment, adult English speakers invented nonsense words as names for random polygons differing in visual complexity. Visually simple polygons received names containing fewer syllables than visually complex polygons did. In addition, analyses of English word-object pairings indicated that a significant correlation between syllable number and visual complexity exists in the English lexicon. In Experiments 2 and 3, adult English speakers matched monosyllabic novel words more often than trisyllabic novel words with visually simple objects, whereas trisyllabic matches were more common for visually complex objects. Experiment 4 replicated these findings with children, indicating that the assumption of a correlation between word and visual complexity exists during the period of intense vocabulary growth. Although the actual correlation between syllable number and visual complexity is small, other posited constraints on word meaning are also limited in strength. However, an increasing number of small, language-specific word-meaning correlations are being uncovered. Given the documented ability of speakers to detect and use these subtle correlations, we argue that a more fruitful approach to word-meaning acquisition would forgo the search for a few broad, powerful word-meaning constraints, and we attempt to uncover individually weak, but perhaps jointly powerful word-meaning correspondences.
\end{abstract}

Throughout the centuries, introspections on the acquisition of word meanings have identified ostensive definition as the prototypical naming game. An adult directs a child's attention toward a certain region of the visual scene while simultaneously muttering some word or phrase. The child then infers that this sound is the linguistic symbol for the relevant feature of the visual scene, and encodes the associated pair into memory. If the child can correctly perform this inference once during every 1 or 2 waking hours between the 1st and 5th years of age, a normal kindergartener's vocabulary will be attained (Carey, 1982).

This apparently simple and intuitively workable naming game has received extreme criticism over the last three decades. Quine (1960) in particular argued that pointing toward an object while speaking a word or phrase does not imply an obvious inference about the meaning of the utterance. For instance, consider a situation in which a

This research was supported by NIH Grant 1 R29 HD23385-01 awarded to Michael H. Kelly and NSF Grant BNS-81-02655 awarded to Frank C. Keil. Thanks are extended to Caroline Fryd-Schechter and Cheryl Pfeiffer for assistance in running Experiments 1-3, and to Michael McCloskey, Arthur Samuel, and three anonymous reviewers for helpful comments on previous drafts of this paper. Correspondence should be addressed to Michael H. Kelly, Department of Psychology, University of Pennsylvania, 3815 Walnut Street, Philadelphia, PA 19104. father points to a glass of liquid while saying to his daughter "water." Even assuming that the child is actually looking at the water rather than the television, her doll, or the family dog, inferring correctly what her father means by "water" would seem to be quite unlikely. The same situation is consistent with other hypotheses, such as that "water" means "container," "clear container," "any clear liquid," "liquid-in-a-glass," "glass-containing-liquid," "currently-unbroken-glass," "daddy's-liquid," or an infinite number of other interpretations. Through timeconsuming experimentation, one could test specific hypotheses, but, as Quine points out, the elimination of some hypotheses would still leave an infinite remainder consistent with the gathered data.

Despite the apparent insurmountability of Quine's (1960) arguments, a critical fact forces one to accept the ultimate feasibility of ostensive definition: It is clearly used between parent and child as well as among adults, and it seems to produce valid inferences about word meanings in the learner. This teaching method is so salient that philosophers as diverse in doctrine as Augustine and Locke have accepted ostensive definition as the obvious method of acquiring word meanings. This observation has steered many investigators away from responding to Quine's dilemma by searching for a radically different approach to meaning acquisition. Instead, researchers have attempted to uncover constraints that human beings 
impose on the types of hypotheses considered in inferring a word's meaning. By sufficiently constraining the nature of these hypotheses, an account of word-meaning acquisition might be proposed that recognizes our extensive use of ostensive definition, as well as Quine's objections to traditional analyses of its implementation.

The types of constraints posited thus far have been quite heterogeneous, carving out different domains of hypotheses about word meanings. However, these constraints are generally cast in the form of assumptions or presuppositions about how languages map onto the conceptual and physical worlds. They are therefore languageneutral, and so they posit assumptions shared by all language learners and users. Markman (1987) has investigated what she calls "the mutual exclusivity" constraint, which rests on the assumption that a single object will not be represented by more than one word (though a single word could map onto multiple concepts, as in /bi/). This constraint is similar to Clark's (1983) lexical contrast theory, although Clark argues that two words may have the same extension, as long as they have different intensions, however slight (e.g., the formality contrast between cop and policeman). In practical terms, the mutual exclusivity constraint implies that if a child has a word for a particular category of objects, then this class will not be considered as a candidate for the meaning of a new word. Children do seem to follow this prediction in making inferences about word meanings. When presented with one object whose name is known and another whose name is unknown, children prefer to pair a novel word with the object currently lacking a name.

Keil (1979) has proposed that children and adults assume that a word's meaning will not conflate different ontological categories. Thus, if a child hears a word paired with a predicate associated with plants, they will then consider as nonsensical or anomalous event predicates paired with the term. For example, when told that "krigias bloom," 5-year-old children accepted the possibility that krigias could die, while at the same time rejecting the possibility that krigias could happen on Saturday (Keil, 1983). On the other hand, when told that krigias could be an hour long, the children accepted "Krigias happen on Saturday" as sensible, but rejected "Krigias die." Children assume that events and plants are nonoverlapping sets, and that word meanings will reflect this division.

Though apparently used by children (and presumably adults) in constraining word-meaning hypotheses, the mutual exclusivity and ontological principles are limited in that the child must already possess a rudimentary vocabulary before they can be applied. Thus, without any word-meaning pairs previously established, the mutual exclusivity principle offers no means of constraining the hypothetical meanings for the first word encountered. Indeed, the constraint would not be very powerful even if a small set of word meanings were known, since few potential meanings for a new word could be eliminated from consideration. In Keil's (1983) ontological studies, children had to be literally given a partial meaning for "krigias" before inferences about other aspects of the meaning could be made. In addition, constraints have generally been discussed as though they were intrinsic to the linguistic domain, rather than deriving from more general cognitive organizing principles. For instance, the mutual exclusivity constraint may reflect a more general assumption that the elements of two domains are mapped in one-to-one correspondence. This relatively abstract assumption may influence cognitive development in areas other than language, such as mathematical reasoning (Gelman \& Gallistel, 1978).

In this paper, we explore a potential constraint that does not depend on the previous acquisition of some word meanings. The constraint that we will discuss possibly derives from a bias to assume that two domains are positively rather than negatively correlated. In other words, when relating two domains, people tend to align the highmagnitude region of one domain with the high-magnitude region of the other domain. For example, subjects in cross-modality matching experiments tend to associate increases in brightness with increases in loudness, and vice versa (Stevens \& Marks, 1965). This association does not depend on the direct perception of brightness and loudness, but can be mediated linguistically. Thus, thunder is rated as louder when it appears in the synesthetic metaphor bright thunder than when it appears in the metaphor dim thunder (Marks, 1982). The adjectives used to describe luminosity in the visual domain can therefore modify the inferences one makes about probable loudness in the auditory domain, and the direction of the inference reflects the assumption that the two domains are positively correlated.

In this paper, we will explore the potential relevance of this general positive-correlation bias to word-meaning acquisition. In particular, we will examine whether adults and children prefer to map visually complex objects onto complex words and visually simple objects onto simple words. Visual complexity will be measured by observer ratings of complexity for familiar and novel designs. The number of syllables in a word will be considered a measure of word complexity, with more syllables corresponding to greater complexity. Given that greater complexity generally entails more difficult processing and concomitant performance deficits, variations in syllable number do appear to be related to variations in processing complexity. For instance, words with many syllables are more difficult to recall from both immediate memory (Baddeley, Thompson, \& Buchanan, 1975) and long-term memory (Bock, 1982). In addition, polysyllabic words appear to be more susceptible to speech errors than monosyllabic words. For example, in a corpus of 183 malapropisms, or one word inadvertently replacing an intended word (e.g., saying "remedy" for recipe), only $26 \%$ involved monosyllabic words (Fay \& Cutler, 1977). Given the fact that monosyllabic words are of relatively high frequency (Zipf, 1965), they would have many more opportunities than polysyllabic words to be involved in a speech error. The dominance of polysyllabic words in such errors sup- 
ports the claim that they are more complex than monosyllabic words. However, it should be emphasized that these complexity effects are not by necessity intrinsically linguistic. Rather, any relatively long motor pattern would in general be more difficult to prepare and produce than a shorter pattern, and any relatively long acoustic signal would in general place greater demands on memory than would a shorter signal.

Given these measures of word and visual complexity, four experiments were conducted to address three questions. First, is there a correlation between the visual complexity of objects and the words speakers invent to denote those objects? Second, do adult listeners map polysyllabic words onto visually complex objects rather than visually simple objects? Finally, do children also assume that complex words will be used to denote visually complex objects? The first question bears on the issue of whether language users are at least partially correct in assuming a correlation between word complexity and visual complexity. This question was addressed in Experiment 1 by presenting adults with drawings of unfamiliar designs varying in complexity and asking them to invent new words for the objects. The basic prediction was that the invented words should consist of more syllables when used to denote visually complex rather than visually simple objects. The second question was addressed in Experiments 2 and 3 , in which adults were presented with paired drawings of familiar or unfamiliar objects. One member in each pair was visually more complex than the other. The subjects were then presented with two words varying in syllable number, and they were asked to pair each word with one of the two objects. Once again, the complexity constraint predicted that the visually complex object would be more likely than the simple object to be paired with the longer word. Finally, the third question was explored by asking children to pair words with objects, with the prediction that visual complexity would be matched with word complexity. ${ }^{1}$

\section{EXPERIMENT 1}

There are two sides to the question of whether or not human beings assume that simple words will map onto simple objects. The first issue concerns the language learner or perceiver, and derives most directly from Quine's (1960) problem. In particular, when a person hears a new word and tries to infer its meaning, are the inferences affected by the complexity of the word? This question focuses on the types of a priori hypotheses that learners or perceivers bring to word-learning situations. The second, equally critical, issue concerns the language producer, and focuses on the validity of word-meaning constraints. A particular constraint on word-meaning hypotheses would not be of help if the lexicon being learned were not governed by the constraint. For example, Carey (1986) has argued, contrary to Keil (1979), that word meanings often conflate ontological categories. Thus, words like Italy possess two meanings that confound the physical object category (a geographical location) and the abstraction category (a government or political system). If such conflations are widespread, then language learning will not be aided, or may even be hindered, by the assumption that the meaning of a word will map onto a single ontological category (see Keil \& Kelly, 1986, for a reply to Carey's critique).

For the complexity constraint to aid the acquisition of word meanings, languages in general, and language speakers in particular, must tend to assign words to objects according to their relative complexities. We first conducted a correlational investigation of this issue by comparing visual complexity ratings for pictures in the Snodgrass and Vanderwort (1980) picture norms with the lengths of their corresponding English words in syllables. These data were collected by the original investigators, and so lexical and pictorial selection could not have been biased by the hypothesis under investigation here. For the 260 pictures used in the norms, we correlated the mean visual complexity ratings for each picture with the number of syllables found in the most common name given to the picture by Snodgrass and Vanderwort's subjects. A small, but significant, correlation of $.20(p<.02)$ was found between visual complexity and syllable number, with number of syllables increasing with complexity.

These results thus indicate that the number of syllables in English words is significantly correlated with the rated visual complexity of their referents. Of course, many potential covariates remain unexplored, so that we have no direct evidence that individual speakers generally tailor their naming habits to fit the complexity of objects named. The correlation is just as consistent with the hypothesis that ratings of visual complexity are affected by the names of the rated objects. In Experiment 1, we addressed these issues by presenting adult English speakers with a set of unfamiliar designs. Subjects were asked to play "Adam," and invent a name for each object. Some of the objects were visually more complex than others, and we explored whether names invented for the complex objects contained more syllables than the names invented for the simple objects. Unfamiliar, randomly constructed designs were used to reduce any effects that might derive from knowledge of the particular objects, such as animacy, prototypicality, or age of learning.

\section{Method}

Subjects. Sixteen members of the University of Pennsylvania community were paid $\$ 2.00$ each for their participation in this 15-20 min experiment. All subjects were native English speakers.

Materials. Randomly constructed, unfamiliar designs were made by modifying slightly a drawing method introduced by Attneave (1957) for studies of visual complexity. The process consists of randomly selecting a specified number of locations on a grid, and then connecting the locations or "dots" by straight lines to create a random polygon. Locations were connected according to proximity, subject to the constraint that lines could not cross, and that the number of edges in the final drawing equaled the number of dots selected. Attneave found that number of edges predicted rated complexity far better than any other variable analyzed (e.g., symmetry). Therefore, in this study, we created two categories of random polygons, 
with 7 or with 15 edges; figures in the latter category were considered to be more complex.

Eight 7-edged and 815 -edged random polygons were created for the study. The figures were created on a Macintosh Plus computer, using the graphics program MacPaint. After the contours of the figures were drawn, the interiors were filled with solid black. Printouts of the figures were used in the experiment, and two examples, one complex and one simple, are shown in Figure 1. The 16 polygons were arranged in two orders, with the first order determined randomly and then reversed to form the second order. Eight subjects were assigned to each order

Procedure. The subjects were told that the experiment concerned the types of rules people use in naming objects. They were asked to imagine that they were modern "Adams" who had to invent names for unfamiliar figures. The names could be anything they liked, as long as the names did not match a known English word. The first figure was then presented, and when the subject had decided on a name, the subject's vocal response was tape-recorded for later analysis. The same procedure was then applied to each of the remaining 15 items.

Scoring and data analysis. The responses were scored by two raters, who were unaware of the complexity of the object named. The raters noted the number of syllables contained in each word, and these were later categorized according to the particular figure being named and its complexity category. The subjects received two scores for each rater: the mean number of syllables used in the names for simple figures, and the mean number of syllables used in the names for complex figures. Items received a single score for each rater, corresponding with the mean number of syllables in the 16 names given to each item. Since all subjects received both simple and complex figures, the means of these categories were compared, using within-subjects tests. However, since items were in either the simple or the complex category, they were compared using between-items tests.

\section{Results and Discussion}

The patterns of significance were identical for the two raters, who agreed in $95 \%$ of their syllable judgments. For ease of exposition, therefore, we will report the results from the rater who showed the smaller difference between the simple and complex figures. As predicted, items in the complex category inspired names containing more syl-

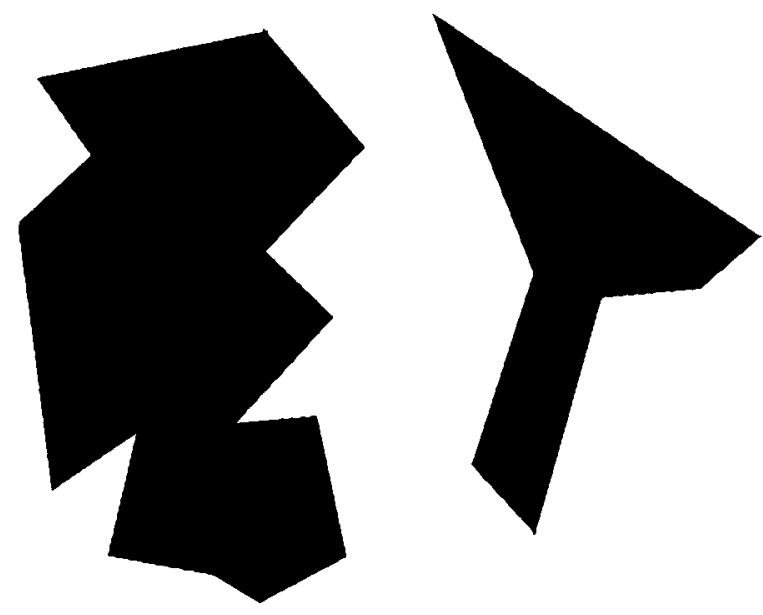

Figure 1. Examples of random polygons used in Experiments 1, 3 , and 4 .
Table 1

Numbers of Simple and Complex Figures That Received Names of Different Syllable Numbers in Experiment 1

\begin{tabular}{lcccccc} 
& \multicolumn{6}{c}{ Number of Syllables } \\
\cline { 2 - 7 } Figure & 1 & 2 & 3 & 4 & 5 & 6 \\
\hline Simple & 57 & 54 & 10 & 5 & 2 & 0 \\
Complex & 31 & 76 & 16 & 2 & 2 & 1 \\
\hline
\end{tabular}

lables than did items in the simple category. The mean number of syllables in names for complex objects was 2.0, whereas the mean for simple objects was 1.8 . This difference was significant across both subjects $[t(15)=2.37$, $p<.05]$ and items $[t(14)=2.32, p<.05]$. Although this difference is small, it should be pointed out that subjects very rarely invented words containing more than two syllables. Only $15 \%$ of all responses contained more than two syllables, and $41 \%$ of these were produced by a single subject. Because of this restricted range of responses, any naming differences between the simple and complex figures would not be expected to be large. To illustrate the differences more finely, Table 1 shows the number of names given to simple and complex figures that contained $1,2,3,4,5$, or 6 syllables. As can be seen, the differences between simple and complex figures are not due to a few outlier responses; they emerge in the distribution of mono- and disyllabic words. In particular, onesyllable words mapped onto simple objects $65 \%$ of the time, but mapped onto complex objects only $42 \%$ of the time. Thus, listeners could make a reasonable inference about the referents of the monosyllabic and disyllabic words invented by the speakers.

When coupled with the correlational analysis discussed earlier, these experimental results indicate that speakers do indeed structure the names of objects in a way that reflects their complexity. Thus, in learning the meaning of a new word, a listener has some justification for assuming that relatively complex words will denote relatively complex objects. It is interesting that the subjects in the naming study never referred to a complexity strategy in explaining how they went about the task. They generally reported giving the first word that came to mind. When told of the complexity hypothesis, they said that it made sense, but that they had not considered this possibility as the purpose of the study. This finding contrasts with the introspections of participants in the following two experiments, in which subjects were presented with nonsense words and selected objects for which the words seemed appropriate names. The subjects frequently mentioned a complexity hypothesis in these experiments. However, the name-invention results suggest that visual complexity is related to word complexity even when this relation is not being explicitly considered at a conscious level. In addition, the appearance of a correlation between syllable number and visual complexity in an extremely unconstrained task attests further to the significance of this relation in mapping words onto meanings. 


\section{EXPERIMENT 2}

Given that languages pair complex words with complex objects to some extent, the next question focuses on whether listeners use this rule as one heuristic when inferring the meaning of a word. In Experiment 2, adult English speakers were presented with pictures of familiar objects arranged in pairs. Within experimental, or target, pairs, one of the pictures was rated more visually complex than the other, with a variety of other factors, such as rated familiarity, controlled. The subjects were told that the objects portrayed are called by different names in different languages, and that they would be presented with two such foreign names for each pair and be asked to guess which word "went best" with each object in a pair. For example, one pair of pictures portrayed an anchor and an arrow, with the latter being rated as less visually complex than the former. The subjects were then given two pseudoword names for the objects, vid and vaniteg, and they had to guess the word that corresponded with each object. In this case, subjects were predicted to pair vid with the arrow and vaniteg with the anchor more than the reverse.

\section{Method}

Subjects. Twenty members of the University of Pennsylvania community were paid $\$ 2.00$ each for their voluntary participation in the 15-20 min experiment. All subjects were native English speakers.

Materials. Twenty-six pairs of pictures were selected from the Snodgrass and Vanderwort (1980) picture norms, with 16 pairs being experimental items and 10 being filler items. These norms were selected because Snodgrass and Vanderwort obtained visual complexity and familiarity ratings for each picture. In addition, since subjects in their study had to name the pictures, we also know the most common name associated with each picture, and hence the number of syllables it contains. The items in each experimental pair differed in visual complexity, but they did not differ in rated familiarity. In addition, the most common English words denoting the objects in each pair contained the same number of syllables, and the frequencies of these words, as determined by Kucera and Francis (1967), did not differ across the simple and complex pictures. Finally, 8 pairs portrayed objects from the same semantic category (e.g., nose and eye), whereas the other 8 portrayed objects from different categories (e.g., arrow and anchor). The simple and complex pictures in the former 8 pairs did not differ in prototypicality, as determined according to the Battig and Montague (1969) category norms. A complete list of the experimental pairs is presented in Table 2. The filler items were selected so that the pictures of each pair were denoted by words containing the same number of syllables. In addition, 5 of the filler pairs portrayed objects from the same semantic category, whereas the remaining 5 pairs showed objects from different categories.

The pictures in each pair were pasted side by side on $3 \times 5$ in. index cards. The left-right order for each pair was determined randomly, subject to the constraint that the visually simple object be placed to the left of the complex object for half of the experimental and filler pairs, and to the right for the remaining half. The cards were then randomly arranged with the restriction that no more than two experimental or filler items occur consecutively.

Sets of 16 monosyllabic, 20 disyllabic, and 16 trisyllabic pseudowords were then invented, with the disyllabic pseudowords assigned to filler pairs and the mono- and trisyllabic words assigned to ex-
Table 2

Object and Pseudoword Pairs Used in Experiment 2

\begin{tabular}{llcl}
\hline Simple Object & Complex Object & Simple Word & Complex Word \\
\hline Star & Tree & Dez & Jalinbaf \\
Envelope & Saltshaker & Gorp & Raminlak \\
Hanger & Glasses & Parm & Dordomak \\
Box & Cake & Dob & Mordinlop \\
Moon & Leaf & Wid & Peninod \\
Arrow & Anchor & Vid & Vaniteg \\
Flag & Crown & Zop & Zoybolam \\
Seal & Lobster & Fot & Tanopee \\
Tomato & Pineapple & Blit & Barodop \\
Ball & Doll & Fim & Ralopor \\
Knife & Gun & Sep & Bovakus \\
Truck & Train & Lish & Saplomay \\
Pig & Skunk & Vorp & Hanlomat \\
Nose & Eye & Gisp & Simlobat \\
Bowl & Stove & Heb & Lakintok \\
Drum & Flute & Yim & Bindolay \\
\hline
\end{tabular}

perimental pairs. One monosyllabic and one trisyllabic pseudoword were randomly selected for each experimental pair, subject to the constraint that the initial phonemes of the pseudowords differ from the initial phonemes of the real English words associated with the objects in each pair. Two disyllabic pseudowords were randomly selected for each filler pair, constrained in the same manner as the experimental pairs. The pseudowords assigned to the experimental pairs are listed in Table 2.

Procedure and scoring. The subjects were instructed that they would play a naming game, in which they would be presented on each trial with drawings of two common objects and a pair of words spoken in a "far-off country." The words were used to denote the objects in that country, and the subjects had to guess which word went with which object. On each trial, the experimenter showed the subject one index card with pictures of two objects. The experimenter would then read the two "foreign" words in a conjunctive phrase (e.g., "gorp and raminlak"), and ask the subject to match the words with the pictures. The experimenter would note the subject's responses on a score sheet, and then begin the next trial.

Both pseudowords used in filler pairs contained two syllables, and the order in which they were spoken was determined randomly. One of the words used in experimental pairs contained one syllable whereas the other contained three syllables. The monosyllabic word was always spoken before the trisyllabic word. This order was selected because English speakers prefer words with few syllables to precede words with more syllables in conjunctive phrases (Bock, 1982; Kelly, 1986), and we wanted to eliminate any possibility that an unnatural presentation of the pseudowords might induce unnatural responses. Since the visually simple object was portrayed to the left of the complex object for half the pairs and to the right of the complex object for the other half, the consistent temporal order of pseudoword presentation was uncorrelated with the spatial order of picture presentation.

For each subject, the percentage of items in which the monosyllabic word was paired with the visually simple object was calculated, and these values were used in the subjects analysis. The scores used for the items analysis consisted of the percentage of subjects who assigned the monosyllabic word to the simple object for each experimental pair.

\section{Results and Discussion}

The results supported the predictions based on the complexity hypothesis, as subjects paired the linguistically simple word with the visually simple object on $65 \%$ of the trials. Across both subjects and items, this asymmetry 
was significantly greater than a chance relationship between word and perceptual complexity [subjects, $t(19)=$ $4.91, p<.001$; items, $t(15)=2.59, p<.025$ ]. Only one of the 20 subjects paired the monosyllabic words with the complex objects more than with the simple objects, and only 3 of the 16 experimental pairs had a higher proportion of subjects making simple-word-complexobject pairings rather than the reverse.

In their introspections on the criteria that they had used to assign words to objects, the subjects often referred to a simple-word-simple-object strategy. However, it is not entirely clear that correctly inferring the hypothesis under investigation is a confound, or whether it provides more direct evidence for the existence of a naturally preferred complexity constraint. Discussions of learning word meanings are typically couched in problem-solving terminology, in which specific hypotheses are formed and tested. Whether these hypotheses are formulated with or without conscious awareness, the fact remains that one can posit many hypothetical correlations between words and meanings. In Experiment 2, for example, the subjects could have decided to match simple words with complex objects. It is certainly logically possible for an inverse correlation between syllable number and visual complexity to exist, and yet not a single subject mentioned this strategy when asked about how the task had been performed. Given sufficient feedback about right and wrong responses, subjects might eventually hypothesize that simple words map onto complex objects in some domain, but this hypothesis seems to be less accessible or reasonable than one relating simplicity to simplicity in word-meaning correspondences. The latter hypothesis emerges without any corrective feedback, which would be expected from a default type of strategy.

\section{EXPERIMENT 3}

In Experiment 3, we attempted to obtain a "purer" test of the complexity hypothesis by using line drawings that did not correspond very readily to familiar objects. Thus, any interference from knowing the English names of objects was reduced, since the line drawings were nonsense figures that lacked English names.

\section{Method}

Subjects. Sixteen members of the University of Pennsylvania community were compensated $\$ 2.00$ each for their voluntary participation in the study. All subjects were native speakers of English.

Materials. Fifty-two random polygons were created, using the procedures described in Experiment 1. Twenty-six polygons contained 15 edges, and were classified as complex, whereas the remaining 26 polygons contained 7 edges, and were classified as simple. The 16 polygons used in Experiment 1 were retained here as part of the complete set. The polygons were randomly arranged into 16 experimental pairs and 10 filler pairs. The experimental pairs contained a 7-edged and a 15-edged figure, whereas the filler pairs contained figures with the same numbers of edges. Thus, 5 of the filler pairs consisted of polygons with 7 edges, and 5 consisted of polygons with 15 edges. The pairs were randomly arranged in top-bottom sequence on standard $8.5 \times 4$ in. computer sheets, under the constraint that half of the experimental pairs have the simple object above the complex object and vice versa for the remaining half.

The 16 pairs of monosyllabic-trisyllabic nonsense words used in Experiment 2 were retained here and randomly assigned to the experimental pairs. The 10 pairs of disyllabic nonsense words used in Experiment 2 were randomly assigned to the filler pairs. The pairs were then randomly arranged for presentation to the subjects, under the constraint that no more than two pairs of the same type appear consecutively.

Procedure. The basic procedure used in Experiment 2 was repeated here, with minor modifications related to the use of random polygons. The subjects were asked to imagine that they had just landed on a new planet, which was furnished with strange objects and whose inhabitants spoke an unfamiliar language. When each pair of objects was presented, the experimenter spoke two words in this language, which corresponded to the names of the objects. The subject was asked to pair each word with the object that seemed to be its most appropriate name. The words in each pair were read in the order used in Experiment 2, and the experimenter noted the subject's responses on a score sheet between trials.

For each subject, the percentage of iterns in which the monosyllabic word was paired with the visually simple object was calculated, and these values were used in the subjects analysis. The scores used for the items analysis consisted of the percentage of subjects who assigned the monosyllabic word to the simple object for each experimental pair.

\section{Results and Discussion}

The results replicated Experiment 2, though the effects were much stronger with the random polygons than they had been with the familiar objects. Subjects matched the monosyllabic nonsense words with the seven-edged simple polygons on $78 \%$ of the trials. This bias to relate word complexity with visual complexity was significant across both subjects $[t(15)=8.35, p<.0001]$ and items $[t(15)$ $=9.93, p<.0001]$. As in Experiment 2, the results were quite robust; not a single subject or item pair showed patterns opposite to those predicted.

As in Experiment 2, subjects often noted the complexity hypothesis when explaining how they tried to perform the task. Though we argue in the discussion of Experiment 4 that such fruitful detective work is not obviously a problem for the complexity hypothesis, and may even be expected, such introspective success raises the question of how powerful the hypothesis is when less constrained situations or less sophisticated human beings are involved. The first study partially addressed this caveat, since the naming task was much less constrained than the matching task, and subjects did not report making conscious use of the complexity hypothesis. Nonetheless, the results support the claim that speakers are biased to encode visually complex with lexically complex patterns. However, all of these studies involved adult subjects, so they do not necessarily reflect the major language-learning task faced in childhood by linguistically naive human beings. In Experiment 4, we examined directly whether or not the complexity hypothesis would be used by children when they inferred the referents of new words. 


\section{EXPERIMENT 4}

\section{Method}

Subjects. Two samples of children ranging in age from 4 to 8 years old were interviewed individually at their day-care centers in the Ithaca, New York area. Each sample was composed of 16 children. The mean ages were 6 years, 10 months for Sample A and 6 years, 4 months for Sample $B$.

Materials and Procedures. The materials and procedures were exactly the same as those in Experiments 2 and 3 involving adult subjects. The children in Sample A matched pseudowords with familiar objects as described for Experiment 2, whereas the children in Sample B matched pseudowords with random polygons, as described for Experiment 3.

\section{Results and Discussion}

The results of both procedures indicated that children, like adults, assume that word complexity is correlated with perceived visual complexity. Sample A matched the monosyllabic word to the visually simple object $60.2 \%$ of the time, a bias that was significantly greater than chance across both subjects $[t(15)=2.51, p<.05]$ and items $[t(15)=2.30, p<.05]$. Only 3 of the 16 subjects paired monosyllabic words with complex objects more than with simple objects, and only 3 of the 16 items received more monosyllabic-complex mappings than monosyllabic-simple mappings.

As in the adult experiments, the results were stronger when the children matched the pseudowords with random polygons. The children in Sample B matched monosyllabic words with the visually simpler, 7-sided polygons on $65.6 \%$ of the trials. This asymmetry was significantly greater than chance across both subjects $[t(15)=4.60$, $p<.001]$ and items $[t(15)=3.91, p<.01]$. Only 1 subject paired monosyllabic pseudowords with 15-edged polygons more often than with 7-edged polygons, and only three items received more monosyllabic-complex matches than monosyllabic-simple matches.

One problem with these results is that they combine data from children of quite different ages. This procedure might occlude important age effects. For instance, if the strength of the complexity bias is positively correlated with age, one might doubt that children follow the bias during the early period of language acquisition. Yet we have argued that the bias might be particularly important in early life because of the relative weakness of other types of word-learning biases. In order to address this issue, we correlated the age of each child in Samples A and B with the proportion of complex word-complex picture matches they made. For the children presented with familiar objects, the Pearson correlation was .048, whereas for the children presented with random polygons, the Pearson correlation was -.408 , with neither of these correlations reaching significance ( $p>.10$ in each case).

In sum, children as well as adults assume that simple words, as defined by syllable number, will denote visually simple figures more often than visually complex figures. Unlike the adults, however, none of the children expressed any explanation even vaguely resembling a complexity hypothesis when asked to justify their responses. This difference suggests that the bias to pair visual stimuli with complex labels may be an early predisposition in the acquisition of word meanings. Since the bias emerged more explicitly among adults, it might be inferred that the complexity hypothesis is an initially unconscious bias that becomes more "accessible" to central processing during cognitive development (Rozin, 1976).

\section{GENERAL DISCUSSION}

When inferring the referent of an unfamiliar word, both adults and children pair complex labels with complex figures. However, the results do not necessarily imply that human beings are predisposed to assume that a specific correlation between word and referent complexity exists. Rather, the results might be a manifestation of a general perceptual/cognitive tendency to align dimensions so that an increase in magnitude along one dimension implies an increase in magnitude along another. This alignment allows people to scale increases in perceived brightness in terms of increases in perceived loudness and, perhaps, increases in perceived visual complexity with increases in perceived label complexity. If correct, then this general explanation for the results reported here should predict analogous effects in structurally similar experiments. For example, one would expect that complex auditory or tactile patterns as well as visual patterns would be related to relatively complex words. (In fact, we have obtained preliminary data consistent with these expectations.)

The complexity heuristic used by language perceivers has some justification, since speakers inventing names for novel figures create words with more syllables when dubbing complex objects than when dubbing simple objects. In addition, a comparison of actual English words with their referents revealed a significant correlation between the number of syllables in the name and the rated visual complexity of the referent. Nevertheless, the correlation between word and visual complexity was quite small, which raises doubts about the power of the constraint, and the wisdom of those who use it. Although the scope of the constraint appears to be restricted, serious limitations plague other proposed word-meaning constraints as well. Thus, both Keil's (1979) ontological constraint and Markman's (1987) mutual exclusivity constraint cannot aid language acquisition until the child already knows the meanings of some words. The complexity constraint is not limited in this way, for it can be used at the start of vocabulary acquisition, although its power nevertheless seems small.

One conclusion we may be forced to draw from this and other work is that few, if any, extremely powerful constraints on word meaning may exist. Rather, human beings may have a large number of heterogeneous constraints, some language-general, others language-specific, but none with any great strength. The correct induction of word-meaning pairs may rely on the joint use of a diverse set of heuristics, whose relevant domains may range from the phonological structure of a word, as with the complexity constraint, to the morphological and syn- 
tactic environment in which a word is embedded. Brown's (1957) seminal discovery that children interpret words with an -ing tagged on the end as denoting actions is an example of a morphologically defined constraint. The "theta criterion," which states that the underlying logical structure of a verb is reflected in its surface argument structure (Chomsky, 1980), is an example of a syntactically defined constraint. Thus, Landau and Gleitman (1985) claim that the meaning of a verb can be inferred from the set of syntactic structures in which it can appear. These constraints are limited in their generality as well. In the Brown example, children must isolate the relevant morphological marker before it can be used as a cue to meaning, but children know the meanings of some words before this point. In order to use the argument structure of a sentence to infer a verb's meaning, one must already be able to differentiate nouns from verbs. The main point is that many quite diverse word-meaning heuristics have been proposed, and actually seem to be used, although they all are basically crutches leaning on other crutches. Thus, an important goal for researchers in language acquisition will be to catalogue these many constraints and determine how they interrelate.

The issue of interrelation could be crucial given that the scope or validity of an individual heuristic is small. If the relation between, say, the syllable number of a word and the perceptual complexity of its referent is of limited validity, why divert attentive and inductive processes to this relation if an infinite number of other possible correlations between linguistic and conceptual structure exist? One possibility concerns the appearance of a particular linguistic variable in other heuristics. Billman (1983) has suggested that learners try to uncover the structure of a domain such as language by searching for variables that appear in many rules. This procedure, called "focused sampling," claims that a particular rule will be uncovered most easily if its components participate in other rules as well. One possible reason for using the low, but present, correlation between syllable number and visual complexity could be the appearance of syllable number in other correlations between linguistic structure and meaning. For example, syllable number is correlated with grammatical category in English, since verbs tend to contain fewer syllables than nouns. Adult English speakers have detected this correlation, and use it when implicitly categorizing nonsense words as nouns or verbs. When asked to use nonsense words such as gorp and raminlak in sentences, speakers are much more likely to use monosyllabic rather than trisyllabic words as verbs (Cassidy \& Kelly, 1990). In addition, syllable number is also correlated with prototypicality, since prototypical members of categories tend to be denoted by words containing relatively few syllables (Brown \& Lenneberg, 1954). Thus, even though the individual correlations between syllable number and the variables of complexity, prototypicality, and grammatical category might be small, their joint existence may direct the attention of language learners and users toward syllable number as a basis for inductions.
Finally, while generally focusing on language-neutral hypotheses about word-meaning relations, investigators may have inadvertently underestimated the role of language-specific word-meaning relations, and the ability of children to detect them. However, a variety of studies have shown that many language-specific cues to a word's meaning or grammatical class exist, and that speakers are amazingly adept at learning and using them. Thus, Kelly (1988) has shown that English speakers have implicit knowledge of the fact that English disyllabic nouns tend to have primary stress on the first syllable whereas disyllabic verbs tend to have stress on the second syllable. Cutler, Norris, and Williams (1987) have demonstrated that English speakers have abstracted the fact that consonant-vowel sequences in English are more likely to have a CVCV rather than CVCC arrangement. Finally, nominal gender categories in French are correlated with certain word endings. Thus, masculine nouns tend to end in -ais whereas feminine nouns end in -ssion. Although not having conscious knowledge of these distributional facts, French-speaking children clearly use them when classifying unknown words into gender categories (Tucker, Lambert, Rigault, \& Segalowitz, 1968). All of these correlations are probabilistic in nature, and, of course, they are not explicitly taught. In addition, they are clearly language-specific. Yet speakers of these languages have a remarkable facility at detecting them, and they can therefore make use of them when they encounter new words. All of this research suggests that many useful correlations between form and meaning exist in language, and, though individually weak, together they could help the child or adult make reasonably quick and accurate inferences about the meanings of novel words.

\section{REFERENCES}

Attneave, F. (1957). Physical determinants of the judged complexity of shapes. Journal of Experimental Psychology, 53, 221-227.

Baddeley, A. D., Thompson, N., Buchanan, M. (1975). Word length and the structure of short-term memory. Journal of Verbal Learning \& Verbal Behavior, 14, 575-589.

Battig, W. F. , Montague, W. E. (1969). Category norms for verbal items in 56 categories: A replication and extension of the Connecticut category norms. Journal of Experimental Psychology, 80, (3, pt. 2).

Billman, D. (1983). Procedures for learning syntactic categories: A model and test with artificial grammars. Unpublished doctoral dissertation, University of Michigan.

Bock, J. K. (1982). Toward a cognitive psychology of syntax: Information processing contributions to sentence formulation. Psychological Review, 89, 1-47.

Brown, R. (1957). Linguistic determinism and parts of speech. Journal of Abnormal \& Social Psychology, 55, 1-5.

Brown, R., \& LENNEBERG, E. H. (1954). A study in language and cognition. Journal of Abnormal \& Social Psychology, 49, 454-462.

CAREY, S. (1982). Semantic development: The state of the art. In E. Wanner \& L. R. Gleitman (Eds.), Language acquisition: The state of the art (pp. 347-389). New York: Cambridge University Press.

CAREY, S. (1986). Constraints on semantic development. In W. Demopolous \& A. Marras (Eds.), Language learning and concept acquisition (pp. 154-172). Norwood, NJ: Ablex. 
Cassidy, K., \& Kelly, M. H. (1990). Phonological bases for grammatical category inferences. Unpublished manuscript.

CномsкY, N. (1980). Rules and representations. New York: Columbia University Press.

Clark, E. V. (1983). Meanings and concepts. In P. Mussen (Series Ed.) and J. H. Flavell \& E. M. Markman (Vol. Eds.), Handbook of child psychology. Vol. 3: Cognitive development (pp. 787-840). New York: Wiley.

Cutler, A., Norris, D., \& Williams, J. N. (1987). A note on the role of phonological expectations in speech segmentation. Journal of Memory \& Language, 26, 480-487.

FAY, D., \& CUTLER, A. (1977). Malapropisms and the structure of the mental lexicon. Linguistic Inquiry, 8, 505-520.

Gelman, R., \& Gallistel, C. R. (1978). The child's understanding of number. Cambridge, MA: Harvard University Press.

GreenberG, J. H. (1966). Language universals. The Hague: Mouton.

KeIL, F. C. (1979). Semantic and conceptual development: An ontological perspective. Cambridge, MA: Harvard University Press.

KEIL, F. C. (1983). Semantic inferences and the acquisition of word meaning. In T. Seiler \& W. Wannenmacher (Eds.), Concept development and the development of word meaning (pp. 103-124). Berlin: Springer-Verlag.

KeIL, F. C., \& KELLY, M. H. (1986). Theories of constraints and constraints on theories. In W. Demopolous \& A. Marras (Eds.), Language learning and concept acquisition (pp. 173-183). Norwood, NJ: Ablex.

KELLY, M. H. (1986). The selection of linguistic options. Unpublished doctoral dissertation. Cornell University.

Kelly, M. H. (1988). Phonological biases in grammatical category shifts. Journal of Memory \& Language, 27, 343-358.

KuČERA, H., \& Francis, W. N. (1967). Computational analysis of present-day American English. Providence, RI: Brown University Press.

Landau, B., \& Gleitman, L. R. (1985). Language and experience. Cambridge, MA: Harvard University Press.

MARKMAN, E. M. (1987). How children constrain the possible meanings of words. In U. Neisser (Ed.), Concepts and conceptual development: Ecological and intellectual factors in categorization (pp. 255287). Cambridge, England: Cambridge University Press.

MaRKs, L. E. (1982). Bright sneezes and dark coughs, loud sunlight and soft moonlight. Journal of Experimental Psychology: Human Perception \& Performance, 8, 177-193.

QUine, W. V. O. (1960). Word and object. Cambridge, MA: MIT Press.

RozIN, P. (1976). The evolution of intelligence and access to the cognitive unconscious. In L. Sprague \& A. N. Epstein (Eds.), Progress in psychobiology and physiological psychology (Vol. 6, pp. 245-280). New York: Academic Press.
SLobin, D. I. (1973). Cognitive prerequisites for the acquisition of grammar. In C. A. Ferguson \& D. I. Slobin (Eds.), Studies of child language development (pp. 175-208). New York: Holt, Rinehart \& Winston.

SNOdgrass, J. G., \& VANDERWort, M. (1980). A standardized set of 260 pictures: Norms for name agreement, image agreement, familiarity, and visual complexity. Journal of Experimental Psychology: Human Learning \& Memory, 2, 174-215.

Stevens, J. C., \& Marks, L. E. (1965). Cross-modality matching of brightness and loudness. Proceedings of the National Academy of Sciences, 54, 407-411.

Tucker, G. R., Lambert, W. E., Rigault, A., \& Segalowitz. N. (1968). A psychological investigation of French speakers' skill with grammatical gender. Joumal of Verbal Learning \& Verbal Behavior, 7, 312-316.

ZIPF, G. K. (1965). The psycho-biology of language. Cambridge, MA: MIT Press.

\section{NOTE}

1. Some related suggestions about predictors of word complexity have appeared in the literature. Thus, Clark (1983) states that "A more complicated word form, for example, 'grandfather' versus 'father,' in general expresses a more complicated idea' (p. 815). However, the investigators she cites as evidence (Greenberg, 1966; Slobin, 1973) do not provide direct support for this claim. Slobin argues that children assume that each conceptual component of a message will have a phonological realization in the morphology of an utterance. However, Slobin does not document the extent to which the child's assumption is always correct. In fact, as evidence for his claim, he points to the fact that children sometimes phonologically mark an unmarked semantic distinction by extending a known morpheme to the unmarked case. Greenberg (1966) focuses on the distinction between marked and unmarked categories in phonology, syntax, and semantics, and offers some examples to suggest that marked concepts are denoted by words with more complex morphology. However, Greenberg's examples are drawn from the kinship category, and no attempt is made to specify the prevalence of the effect in other conceptual domains. (It is ironic in this regard that the English word for the general concept of not marked has a more complex morphology ["unmarked"] than does the English word for the general concept of marked [i.e., "marked"].)

(Manuscript received May 9, 1988; revision accepted for publication January 1, 1990.) 